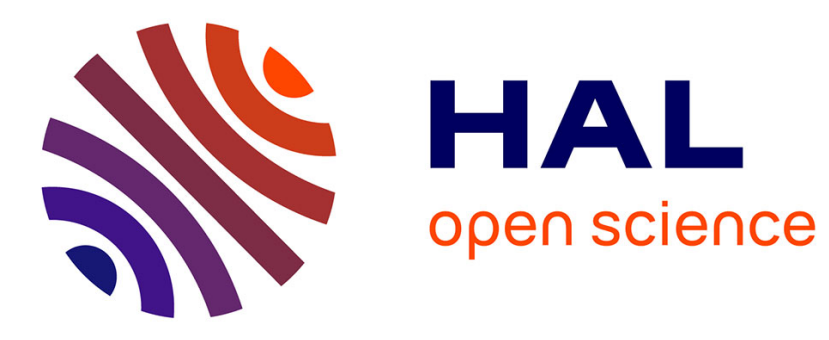

\title{
Influence of Thermal Effects During Blade-Casing Contact Experiments
}

Antoine Millecamps, Jean-François Brunel, Philippe Dufrenoy, François Garcin, Marco Nucci

\section{To cite this version:}

Antoine Millecamps, Jean-François Brunel, Philippe Dufrenoy, François Garcin, Marco Nucci. Influence of Thermal Effects During Blade-Casing Contact Experiments. ASME 2009 International Design Engineering Technical Conferences and Computers and Information in Engineering Conference, Aug 2009, San Diego, United States. 10.1115/DETC2009-86842 . hal-01223060

\section{HAL Id: hal-01223060 https://hal.science/hal-01223060}

Submitted on 7 Sep 2016

HAL is a multi-disciplinary open access archive for the deposit and dissemination of scientific research documents, whether they are published or not. The documents may come from teaching and research institutions in France or abroad, or from public or private research centers.
L'archive ouverte pluridisciplinaire HAL, est destinée au dépôt et à la diffusion de documents scientifiques de niveau recherche, publiés ou non, émanant des établissements d'enseignement et de recherche français ou étrangers, des laboratoires publics ou privés. 
Proceedings of the ASME 2009 International Design Engineering Technical Conferences \& Computers and
Information in Engineering Conference
IDETC/CIE 2009

DETC2009/VIB-86842

\section{INFLUENCE OF THERMAL EFFECTS DURING BLADE-CASING CONTACT EXPERIMENTS}

\author{
Antoine Millecamps \\ Laboratoire de Mécanique de Lille \\ Boulevard Paul Langevin \\ 59655 Villeneuve D'ascq, France
}

\author{
Jean-François Brunel \\ Laboratoire de Mécanique de Lille \\ Boulevard Paul Langevin \\ 59655 Villeneuve D’ascq, France
}

\author{
Philippe Dufrénoy \\ Laboratoire de Mécanique de Lille \\ Boulevard Paul Langevin \\ 59655 Villeneuve D’ascq, France
}

\author{
François Garcin \\ Snecma Villaroche \\ Rond Point René Ravaud-Réau \\ 77550 Moissy-Cramayel, France
}

\author{
Marco Nucci \\ Snecma Villaroche \\ Rond Point René Ravaud-Réau \\ 77550 Moissy-Cramayel, France
}

\begin{abstract}
In rotating machinery, notably in modern high efficiency compressors, a critical requirement for optimal performance consists in minimizing radial clearances between the rotating bladed disk and the casing. This solution significantly increases the risks of contact between rotating bladed disk and casing and may lead in specific conditions to catastrophic behavior (component failure, etc.). The physical phenomena and mechanisms involved in blade-casing contact interaction situations are still misunderstood. In order to highlight these mechanisms, specific experiments have been performed on an experimental multi-stage compressor of a turbojet with dedicated dynamic and thermal instrumentations. For all configurations tested, major damages are noticed: blades had cracks and the abradable coating of the casing was heavily machined. Results show that the blade failure refers to fatigue limit with first natural mode excitation of the blade. The paper is focused on the analysis of the successive stages of blade dynamic response before the failure. It is shown that this response is influenced by the variations of the blade-casing contact conditions. These conditions are linked to the thermomechanical behavior and wear of coating, illustrated by high thermal levels and non uniform wear profile. Coupling between thermomechanics, wear and dynamic has to be considered to highlight the transient mechanisms leading to the cases of blade failure.
\end{abstract}

\section{INTRODUCTION}

The phenomenon of contact between the rotating bladed disk and the surrounding casing becomes a design factor for today's turbo machinery compressors. The search for a high compression ratio with a small number of stages (weight savings/lower cost) requires the adoption of smaller rotor/stator clearances at the blade tip: the front/back pressure difference creates turbulence, which increases with the stage load. These leaks lead to loss of aerodynamic performance and of compressor surge margin. The use of extremely tight clearances is a way to control with these phenomena. With these new architectures, the risk of contact between the rotating bladed disk and the casing increases significantly. These contacts may occur in conventional operating conditions (engine ageing, operating load, slight out-ofbalance, running-in, re-acceleration, etc.), or extreme conditions (particular aerodynamic and thermal operating point). Transient loads generated by these rotating bladed disk-casing contacts can lead to cyclic bending of the blade and to its damage by fatigue cracking. The problematic is that the mechanisms involved in this damage configuration are not yet well understood.

In the past, the scientific community performed a limited amount of work concerning the non-linear dynamics generated by this type of interaction [1-7]. Most works in this field concern the study of a cylindrical shaft type rotor which comes into contact with a stator with a circular interior contour. The studies concerning a bladed type rotor are far rarer. Moreover, it must be accepted that none of this work has yet given a reliable understanding of the dynamics generated at contact. The main reason is that the physical mechanisms involved are both multi-physical [8] (vibrations, rubbing, heating, wear, etc.) and multi-scale [9] (coupling of dynamic and thermomechanical responses in the contact zone and in the structures, with frequently complex geometry). Moreover, the contact is a very complex mechanism, since the casing is covered with an "abradable" coating applied by thermal spray techniques and containing a phase material, with high porosity, offering a good balance between abradability and erosion resistance [10-13]. Its role is to be worn when an interaction has happened, to protect blades and to obtain a 
smooth surface after an interaction. Unfortunately, damages on bladed disk are still noticed although a coating is laid.

Two classes of research works on the bladed disk/casing contact can be distinguished: the dynamic analysis of rotor/stator system, with numerical models development to simulate the nonlinear dynamic behavior of the rotor/stator assembly by transient simulation or nonlinear analysis [14]; experimental investigations of the local interaction between an abradable material and a blade to determine contact forces and wear mechanisms [15]. The dynamic behavior does not seem to be sufficient to explain the interaction occurrence. Moreover, at the local level, experimental measurements may show high temperature elevations. The contact seems to correspond to an extensive coupling existing between the overall dynamics of the system and the physical phenomena occurring at the local contact friction surface and involving the material and the tribological characteristics of the materials (erosion of the abradable coating, etc.). The relation between these mechanisms of the contact and the dynamic behavior has to be improved. This is the aim of this paper with an experimental analysis of the influence of the thermomechanical couplings on the dynamic blade response. Specific experiments have been investigated on a low pressure turbojet compressor with a dedicated instrumentation. The paper is divided in 5 sections. After this introduction, the experimental set-up is detailed in Section 2. Section 3 is devoted to the analysis of the blade dynamic response. The influence of thermomecanical couplings is discussed in section 4. Finally, the paper is concluded in Section 5.

\section{EXPERIMENTAL SET-UP}

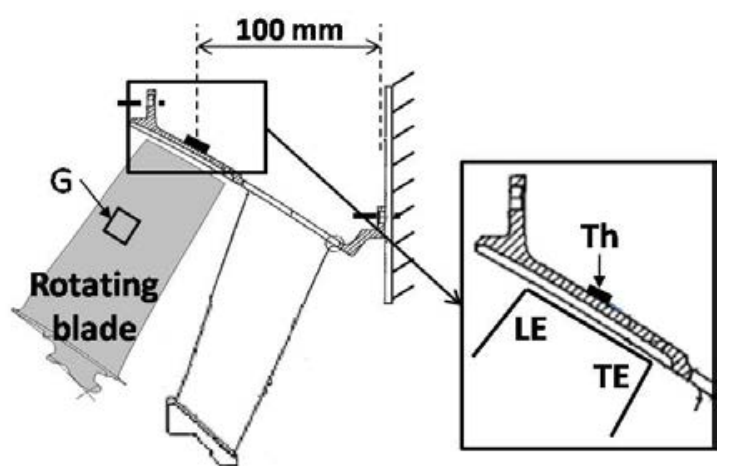

\footnotetext{
- 所- - - - - - -

Figure 1. Cross section of the compressor tested with the stationary blades of casing in white. Zoom of the casing with the thermocouples position and the definition of Leading Edge (LE) and Trailing Edge (TE). (G) represents, in broad outline, the position of the strain gage on suction side.
}

Blade-casing contact tests are performed on an experimental multi-stage compressor of a turbojet on a same scale as that of the practical turbomachinery rotor and under vacuum to limit rotation, temperature and aerodynamic loadings. Stages are screwed together and the last stage of compressor is screwed on a heavy frame (figure 1). The stage is covered by an abradable coating facing the blade. Only the rotor of the last stage has a bladed disk, driven by an electrical motor.

The bladed disk has one blade longer than the others in order to localize the blade-casing interaction on this blade. The longer titanium blade has one strain gage positioned on suction side sensitive to the bending motion of blade. The titanium casing with its abradable coating is equipped with thermocouples. Their numbers depend on the test configuration and they are located at the outer side, at $100 \mathrm{~mm}$ from the frame (figure 1). A sensor measuring the clearance indicates a blade-casing contact.

Two configurations have been particularly focused. In the first one, the rotor speedup aims at reaching a critical rotational speed in order to excite the blade on one of its natural frequencies (figure 2). The second configuration aims at forcing the blade rubbing on the casing using centrifugal loads (figure 3). For the two configurations, once objectives of the test are obtained, the rotational speed is kept constant. The first configuration is performed by step-by-step speed increases. In the second configuration, the first step has been performed to close clearances and then a quick acceleration is applied.

Note that for the two configurations, the abrabable coating and the blade geometry are different.

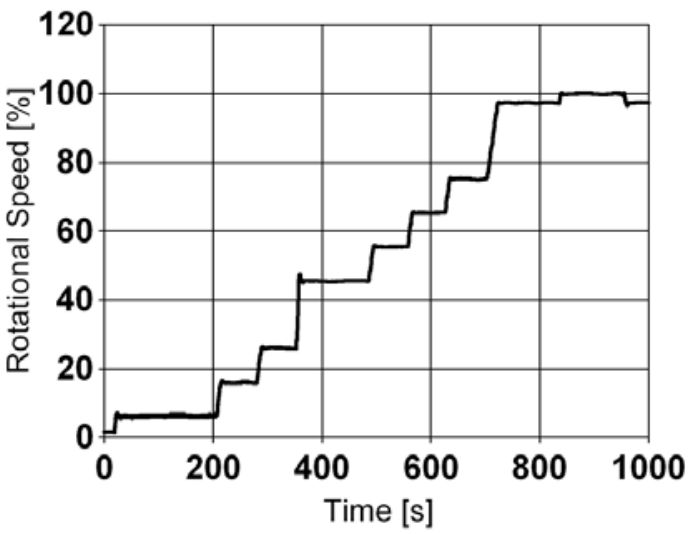

Figure 2. Rotational speed in step-by-step for the first configuration.

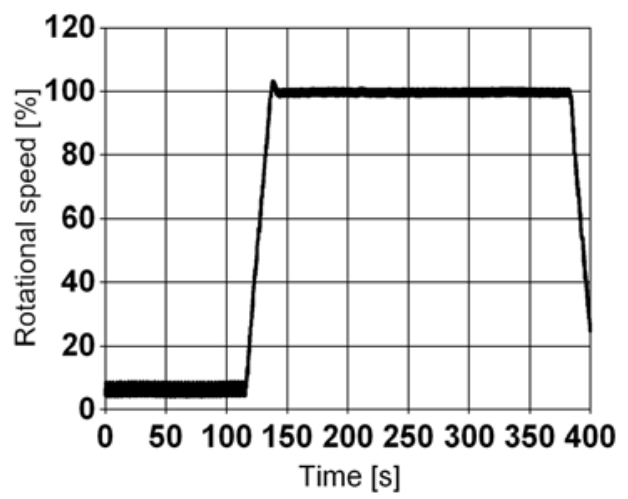

Figure 3. Rotational speed for the second configuration.

For each configuration, the natural frequencies of blades have been determined using numerical modal analysis, assuming a clamped dovetail of blade and no aerodynamic loadings. The analysis takes the centrifugal stiffness into account to compute 
the natural frequencies as a function of the rotational speed. The first bending and twisting mode frequencies are determined and are plotted on the Campbell diagram (figure 4). In the first configuration, the first bending mode frequency is an integral multiple of the rotation speed (ratio of 7). Note that the first twisting frequency is also an integral multiple of the rotation speed (ratio 25). For the second configuration, the ratio between the speed and the first natural frequency is 5.5.

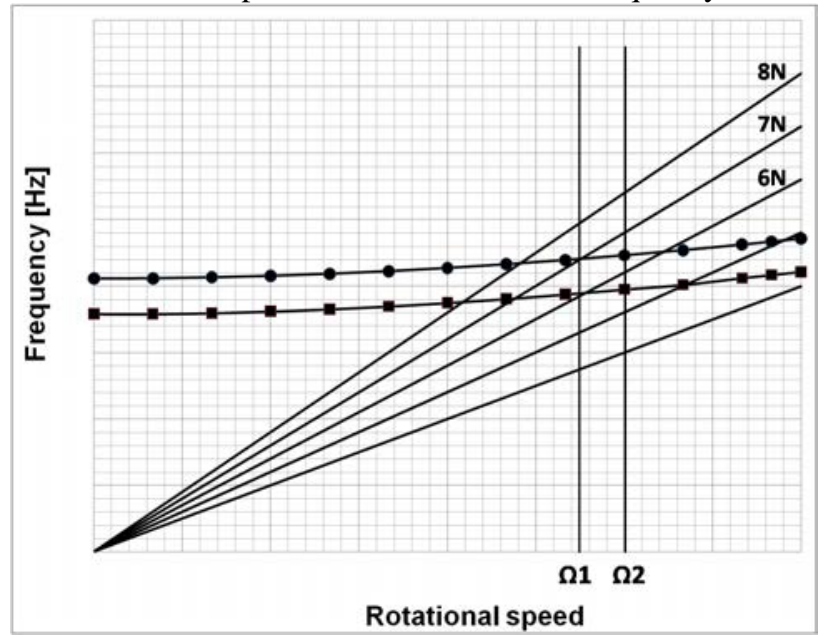

Figure 4. Natural frequencies of the blades of configuration $1(\bullet)$ and the blades of configuration $2(\mathbf{})$.

$\Omega 1$ is the rotational speed of configuration 1 and $\Omega 2$ is the rotational speed of the configuration 2 .

An important point is that the casing is not particularly circular due to mounting conditions. An ovalization of the casing has been observed for the two configurations. The second configuration leads to more constrained blade-casing contact conditions, according to configuration objectives.

Each case led to same observations: the longer blade had cracks and the abradable coating was heavily damaged at the end of the tests. In the following, blade vibration response is analyzed for each configuration.

\section{ANALYSIS OF THE BLADE DYNAMIC RESPONSE}

Post-mortem observations for the first configuration show that the longer blade had cracks near the dovetail due to fatigue and that its leading edge was curled-up and that its dovetail was damaged. The blade stress response for the first configuration is plotted in figure 5, from $800 \mathrm{~s}$ to the end of the test when the longer blade had failure.

The time variation of the blade stress $(\sigma)$ is divided by the absolute value of the stress due to the centrifugal loads $\left(\sigma_{\omega}\right)$. The stress $\sigma_{\omega}$ is the value recorded by the gage as soon as the steady-state is reached. Note that $\sigma_{\omega}$ is negative due to the specific geometry of blade. Due to its complex shape and submitted centrifugal loads, the deformation measured by the gage and used to calculate stresses assuming a linear elastic material behavior, is negative at this location (figure 1).

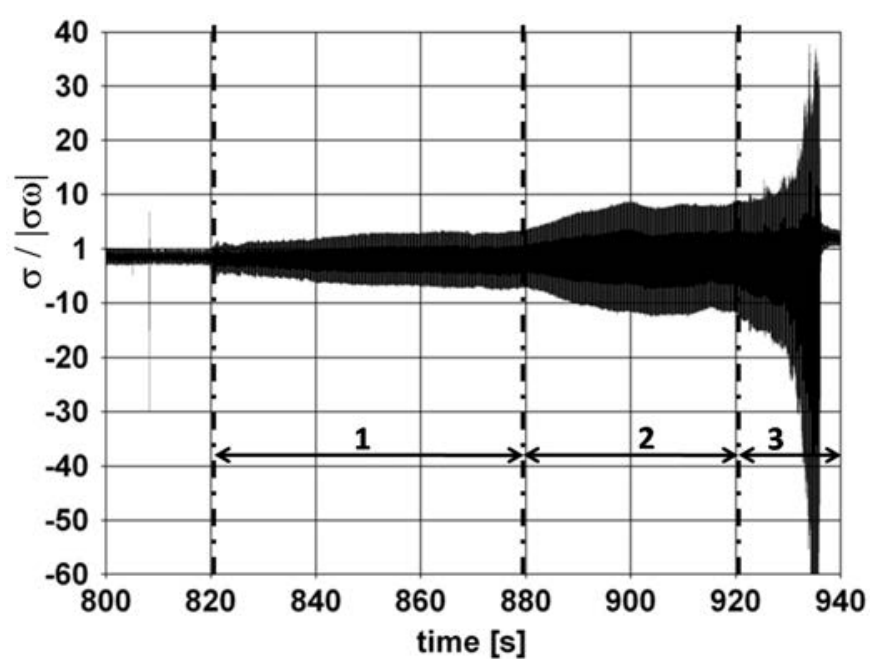

Figure 5. Blade stress time variation during the rotational speed steady state of the first configuration.

Three phases may be distinguished from the blade stress signal: the first one begins as soon as the steady state is reached (at 820 s). Note that very low blade stress time variations were recorded before this time, resulting in no blade-casing contact. At $820 \mathrm{~s}$, blade-casing clearances were closed and the amplitude of longer blade stress slowly increased in the beginning of the first phase and then stabilized during a step more than $20 \mathrm{~s}$ up to 880 s. In a second phase (between 880 to $920 \mathrm{~s}$ ) same phenomena were recorded with maximum stress amplitude reaching approximately 5 times $\sigma_{\omega}$. After almost $20 \mathrm{~s}$ of increasing, the stress amplitude remained close to the same value during more than $20 \mathrm{~s}$. Note that the rotational speed remains the same during the two phases. The third phase begins at $920 \mathrm{~s}$ when the blade stress amplitude increased continually up to the blade stress signal is lost, probably due to blade cracking (at time around $935 \mathrm{~s}$ ). After this time, the test was stopped.

To complete the analysis of blade stress time-variation, harmonic analyzes for one revolution were performed according to the identified phases previously described. The two first phases were characterized by a complex stress time variation with two major modes contribution: harmonics 7 and 25 of the rotational speed, referring to the first bending and twisting natural frequency of the longer blade (figure 6). The blade had a combined bending-twisting resulting motion.

During the last phase, the blade had a vibratory response with 7 periods per revolution (figure 7) Note that the blade stress time variation for two revolutions (at 900 and 915 s) are plotted in figure 7 , showing less phase difference between the two revolutions. The blade motion seems to be accorded with the harmonic 7 of the rotational speed leading to its failure. 

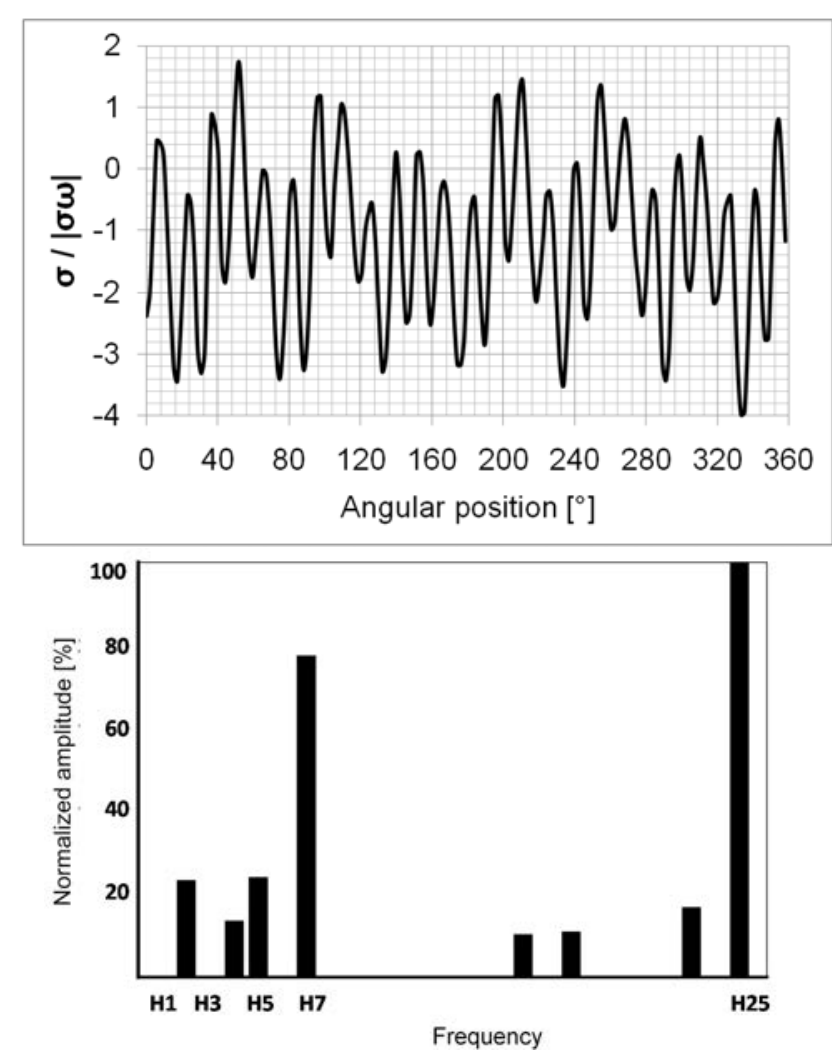

Figure 6. Variation of the blade stress during one revolution of the first phase at $850 \mathrm{~s}$ and its harmonic content.
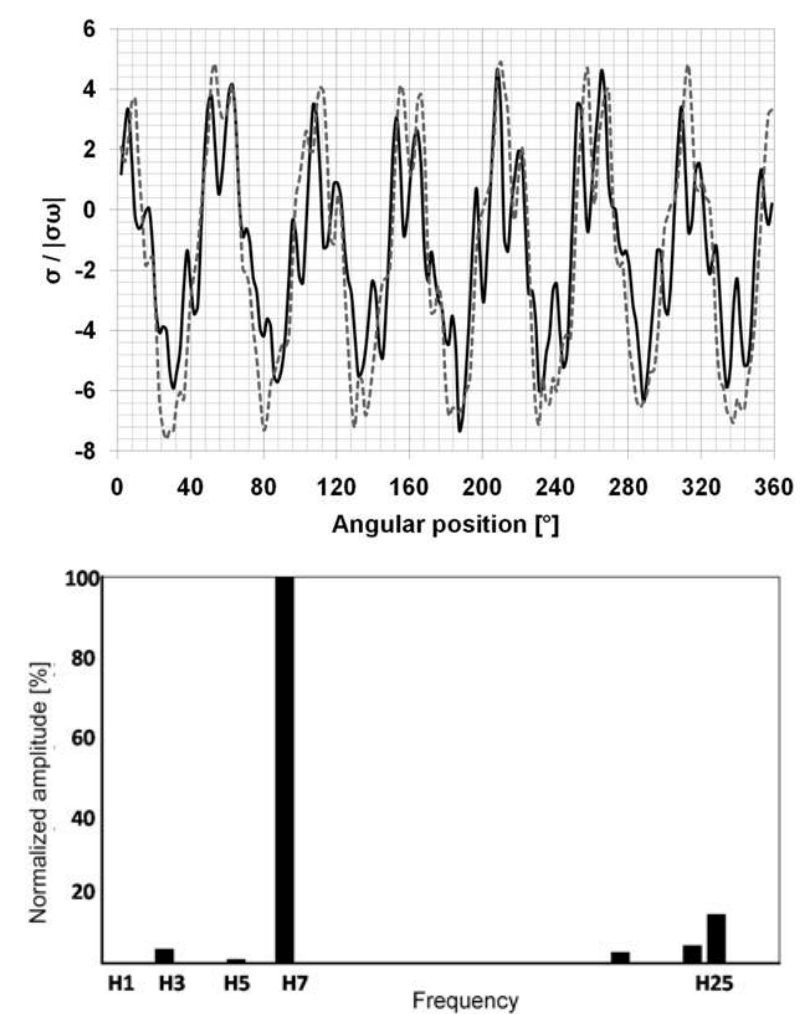

Figure 7. Variation of the blade stress during two distinct revolutions, at $900 \mathrm{~s}$ and $915 \mathrm{~s}$, of the third phase and the harmonic content of the bold curve.
Same analyses were performed for the second configuration which objective is to force the blade rub using the centrifugal loads (figure 3 ). This configuration test has led to a divergence of the blade too. The blade had cracks and its dovetail and its trailing edge were damaged. The longer blade had very locally a change of color due to an overheating at its tip. The temperature of the whole longer blade does not seem to vary significantly as the heating is only due to the frictional dissipation at the blade-casing contact. It probably does not affect the dynamic characteristics of the blade. The blade stress amplitude time-variation is plotted in figure 8 . The time variation of the blade stress $(\sigma)$ is also divided by the absolute value of the stress due to the centrifugal loads $\left(\sigma_{\omega}\right)$. Like in the previous configuration, the blade stress amplitude variation shows several phases. Note that during the first part of the test a blade-casing contact has been detected. The analysis is focused on the second part of the test after the quick acceleration: 5 phases may be distinguished. Several unstable behaviors may be observed on the blade stress amplitude timevariation (phase 2, phase 4 and phase 5). The last phase leads to the longer blade failure. For the two first unstable phases, amplitudes suddenly decrease.

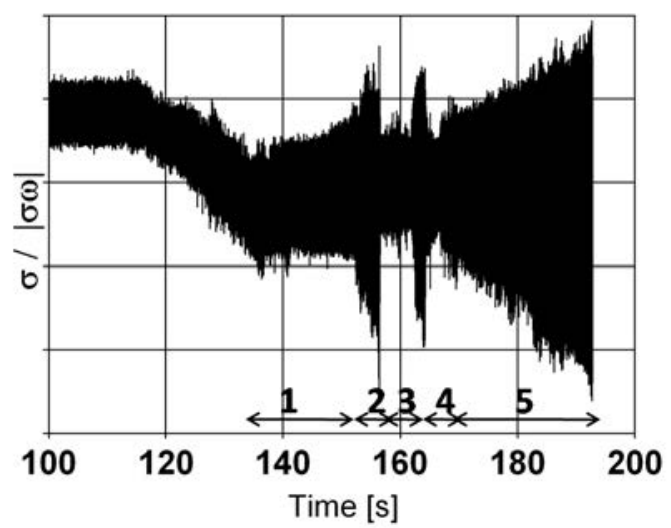

Figure 8. Blade stress variation from $100 \mathrm{~s}$ to $200 \mathrm{~s}$ during the steady state of the second configuration.

During the first phase (from $138 \mathrm{~s}$ to $155 \mathrm{~s}$ ), time variation of the blade stress during one rotor revolution show mainly a sine signal with two periods. Many frequencies are excited even if a 2 multiple of the rotational speed (H2) is more significant (figure 9). Harmonic 4 is also observed at the beginning and H5, H6 and H7 slightly appear (figure 9).

The second phase is characterized by a complex unstable blade stress behavior with a predominance of $\mathrm{H} 7$ and the notable apparition of $\mathrm{H} 5$ and $\mathrm{H} 2$ (figure 10).

During the third phase, from $158 \mathrm{~s}$ to $162 \mathrm{~s}$, no more sensitive activity is noted; the level of blade vibratory response is very low. Then the fourth phase is similar to the second one. The amplitude of blade stress is notably increasing but it suddenly decreases, as at the end of phase 2.

During the fifth phase the blade has an unstable behavior leading to its failure. The amplitude is increasing more and more until the blade is damaged at $190 \mathrm{~s}$. Lastly the fifth phase shows clearly that $\mathrm{H} 6$ is the most predominant frequency (figure 11); the amplitude is strongly increasing leading to the blade divergence and to its failure. A focus on one revolution of the blade stress during the fifth phase shows 6 flexions per rotor cycle, as illustrated in figure 11 at two distinct 
revolutions. No phase difference between two variations of stress plotting is observed.

The analysis of dynamic data of two configurations which objectives are different shows very complex blade stress timevariation induced by blade-casing contact. None of the configurations led to immediate unstable behavior, several stages are generally observed with long variation compared to the rotational frequency with various types of dynamic responses. For the second configuration, it seems that unstable behaviors initiate and suddenly stop separated by phases of low stress amplitude. It has been shown that for the two configurations, blade failure is the consequence of the excitation on the first bending mode of the blade with a frequency very close to its natural value. For all the configurations, no coincidence of vibrations modes between the casing and the bladed disk was deduced. However such dynamic analyses are not enough to explain all the successive phases observed before the unstable blade dynamic response. In the following, thermomechanical behavior of blade-casing contact is analyzed in terms of abradable wear and thermal measurements in order to clear the study of blade-casing contact.
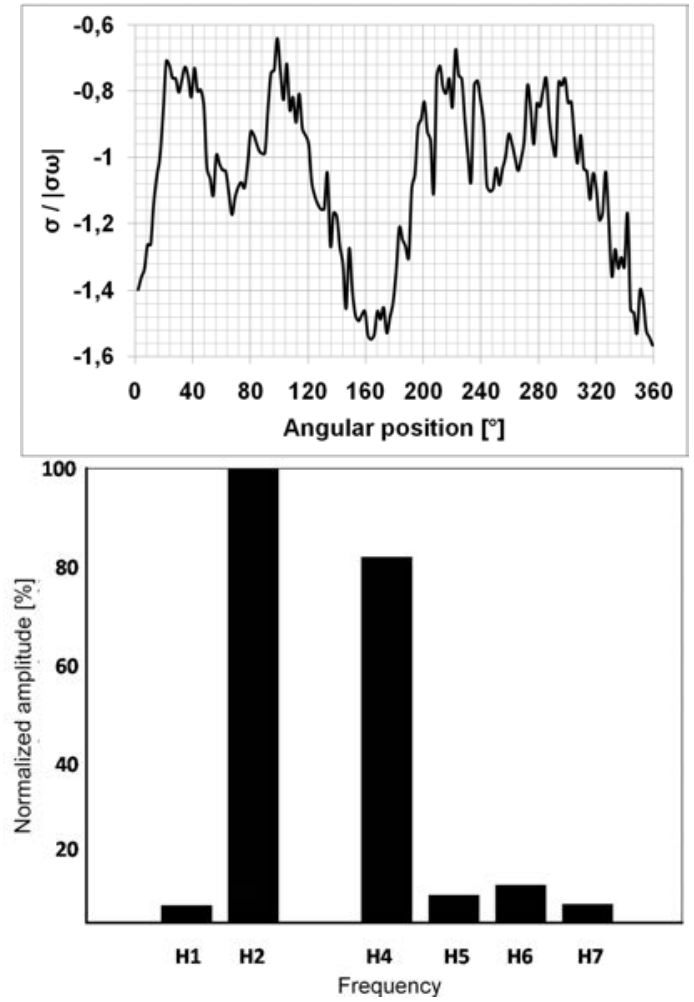

Figure 9. Time variation of the blade stress during one revolution of the first phase and its harmonic content.
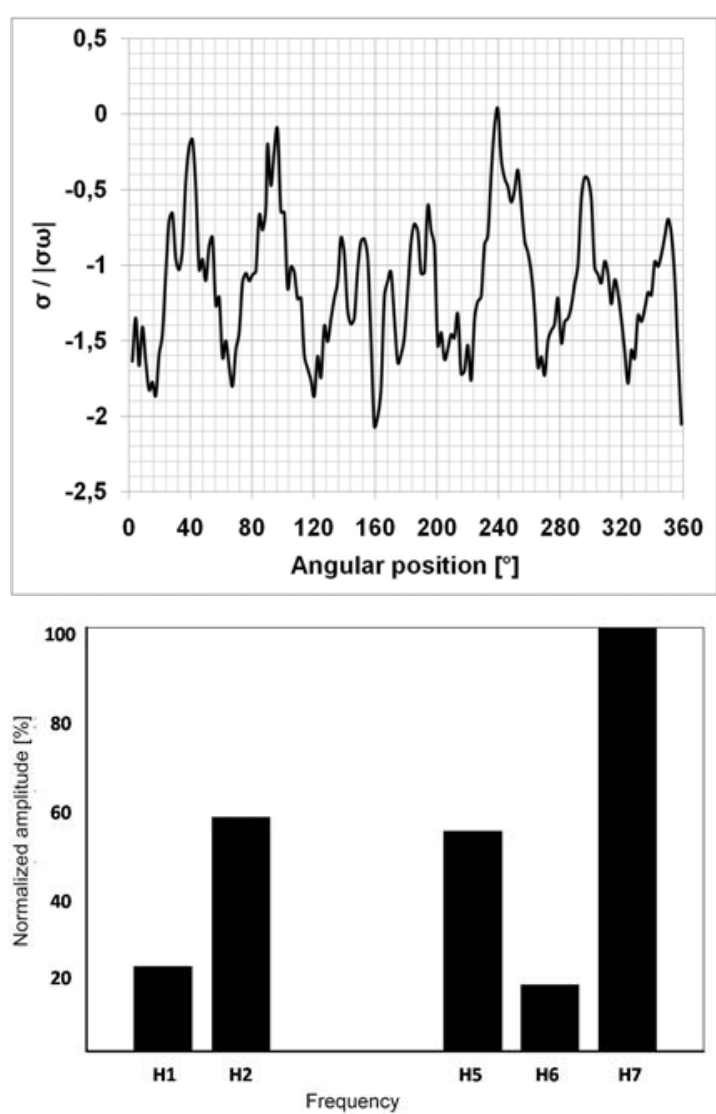

Figure 10. Time variation of the blade stress during one revolution of the second phase and its harmonic content.
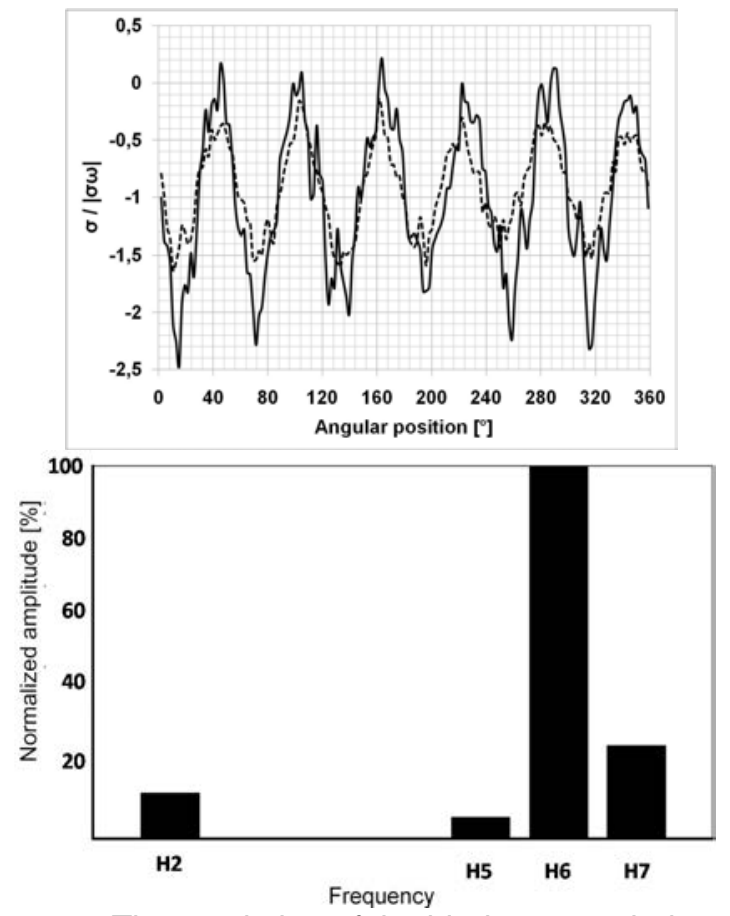

Figure 11. Time variation of the blade stress during two distinct rotor cycle (at $176 \mathrm{~s}$ and $190 \mathrm{~s}$ ) of the last phase and the harmonic content of signal plotted in bold. 


\section{ANALYSIS OF THERMOMECHANICAL DATA 4.1 Analysis of abradable wear}

In addition to blade damages, the wear of abradable coating is significant even if it is post-mortem observations after the experimental configuration dismantle. In various places the metal of casing can be seen while the abradable coating is new at certain location. For the two configurations, the coating wear is not uniform in the circumferential and axial directions. Figures 12 and 13 show the wear profile along the casing circumference at two axial positions (facing the leading edge and the trailing edge) for the two configurations previously described.

In the first configuration, the circumferential wear facing the leading edge of the blade has a prominent 2-lobes wave with a depth of almost $0.46 \mathrm{~mm}$ (figure $12 \mathrm{~b}$ ). The main wear is at $0^{\circ}$ and $180^{\circ}$ coinciding with the initial ovalization of the casing. Since the clearance is smaller, wear is higher. The circumferential wear facing the trailing edge of the blade has a prominent 7-lobes wave (figure 12a). In places, the wear is low. The most important wear depth is $3 \mathrm{~mm}$ (locally the coating is completely consumed).

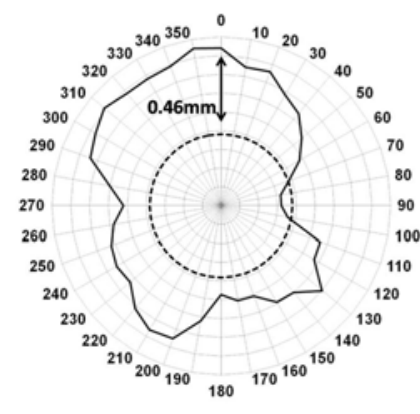

(a)

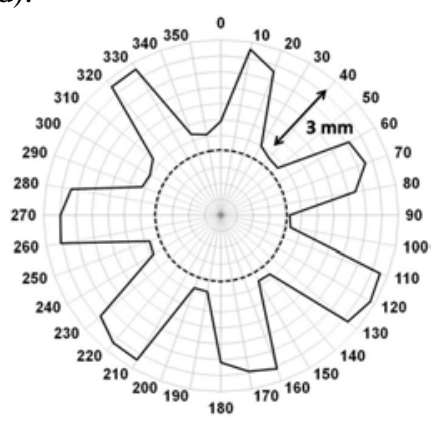

(b)
Figure 12. Circumferential wear of the abradable coating, at two different axial locations, facing the LE of blade (a) and facing the TE of blade (b) in the first configuration. Dashed circles are the initial radius.

In the second configuration, the abradable coating is heavily machined. Like in the first configuration its wear is not uniform depending on the circumferential and axial locations. The circumferential wear at axial location facing the leading edge (figure 13b) has a prominent 2-lobes wave with a depth of $1.5 \mathrm{~mm}$. The main wear is at $0^{\circ}$ and $180^{\circ}$ corresponding to an initial ovalization of the casing. The circumferential wear at axial location facing the trailing edge of the blade has clearly a prominent 6-lobes wave (figure 13a). The depth of the higher lobe is approximately $3 \mathrm{~mm}$, the abradable is totally consumed. In some places, the coating is almost new.

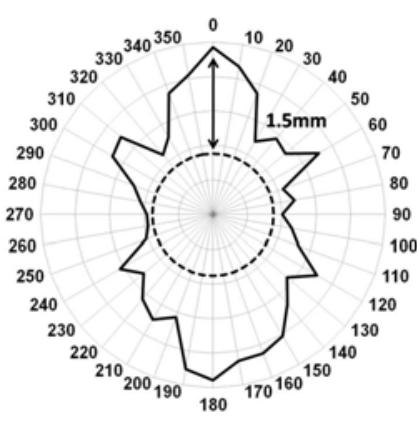

(a)

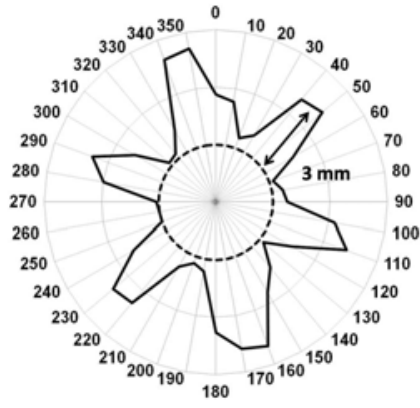

(b)
Figure 13. Circumferential wear of the abradable coating, at two different axial locations, facing the LE (a) and facing the TE (b) in the second configuration.

Dashed circles represent the initial radius.

For the two configurations, the abradable coating wear profile strongly depends on the axial location. The abradable facing the leading edge is quite less machined with a prominent 2lobes wave whereas the circumferential wear facing the trailing edge features a profile with six or seven lobes. The contact between the blade and the casing is not uniform and clearly stronger at the trailing edge.

An important result is that the circumferential wear has a multi-lobes wave. Depending on the test configuration, the abradable coating worn by the trailing edge of the blade features a profile with 6 or 7 lobes. In the previous paragraph we observed at the end of each test unstable blade behaviors with six or seven bending motion per revolution. In the first configuration, the blade response is a 7-bending motion per revolution during its unstable behavior. The circumferential wear has a 7-lobes wave. In the second configuration, the circumferential wear has a prominent 6-lobes wave while the blade has an unstable behavior with a 6-bending motion. The number $\mathrm{N}$ of main lobes shape corresponds to the ratio between this mode and the rotational speed giving $\mathrm{N}$ flexions of the blade per revolution. So the abradable wear and the dynamic response of blade are strongly linked. In the following we study the relation between the abradable wear and the blade dynamic for the first configuration since phenomena are exactly similar for the second configuration.

The wear of coating facing the trailing edge has a prominent 7-lobes wave and the last phase of blade dynamic response is a 7-bending motion. A comparison of the abradable wear shape and the dynamic response of blade during one revolution is shown in figure 14. According its location, the gage is sensitive to the bending motion and a maximum corresponds to a bending toward the pressure side. Similarly a minimum means a blade bending toward suction side. Stress seems to reach a maximum at the same circumferential location as wear lobes. It seems that abradable coating wear occurs when the blade is bending toward its pressure side. Same correlation is observed for all revolutions during the unstable vibratory response.

The coating wear profile facing the trailing edge of blade can be correlated to the dynamic response of the longer blade. Rub depths are consistent with the first bending mode dynamic response seen on gage measurements. 

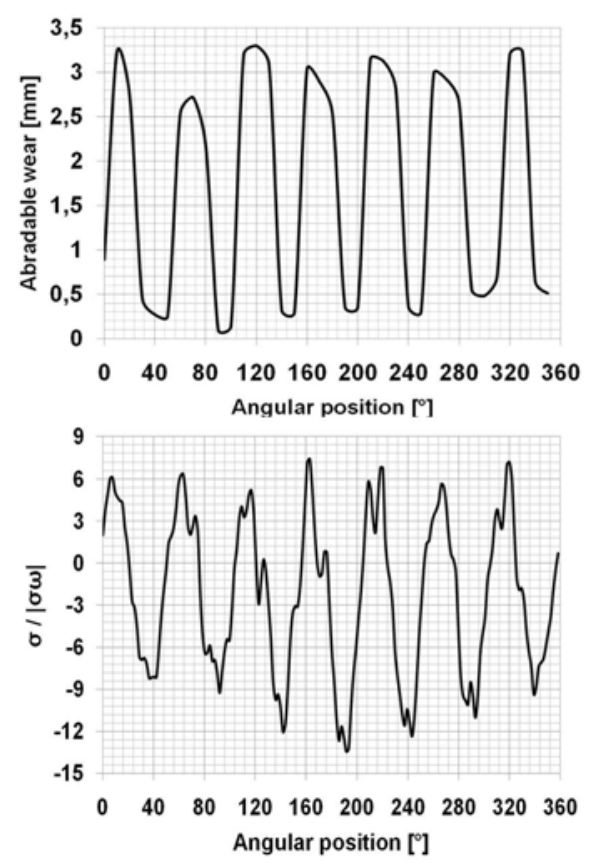

Figure 14. Circumferential wear of coating facing the trailing edge (at $86 \mathrm{~mm}$ ) and underneath the variation of blade stress during one revolution of the third phase of the first configuration.

\subsection{Analysis of thermal data}

Analysis of thermal data from the first configuration is detailed in the following thanks to high number of thermocouples, which is not the same for the second configuration. 9 thermocouples are located on the outer side of casing at different circumferential locations as shown in figure 15. The axial location of the thermocouples is shown in figure 1.

Temperature recording during the test (figure 16) shows that significant temperature increases were recorded for the thermocouples located at $210^{\circ}$ and $318^{\circ}$ after the blade has a mainly bending motion (corresponding at the beginning of phase 2 of figure 5). Note that the temperature at the bladecasing contact is certainly much higher as the low thermal conductivity of the abradable coating attenuates the thermal level at the rear of the casing, where the thermocouples are located.

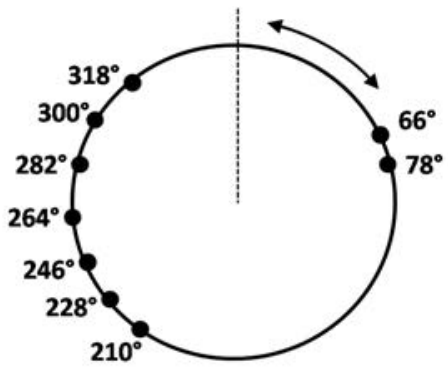

Figure 15. Circumferential position of thermocouples. Casing is seen from trailing edge to leading edge.

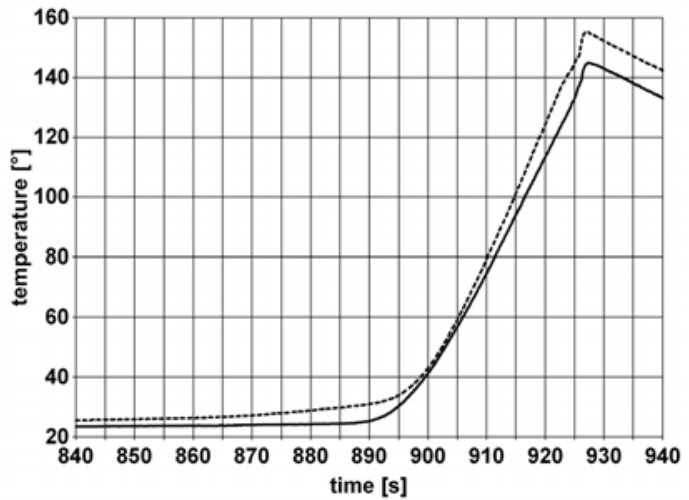

Figure 16. Temperature of thermocouple located at $318^{\circ}$ (full line) and $210^{\circ}$ (dashed line).

Figure 17 shows maximal temperature variation obtained during the test on the graph of circumferential wear of casing. This graph differs from the previous ones in figure 12 since it is plotted at the same axial location of thermocouples, at 100 $\mathrm{mm}$ from the frame. It appears that the thermocouples facing a lobe of wear (located at $66^{\circ}, 210^{\circ}, 264^{\circ}$ and $318^{\circ}$ ) shows a significant increase of temperature while the others reveal no variation. Even if few increases are low we can observe that thermocouples which do not face a lobe have no thermal variations. Again here the low conductivity of the casing may explain low propagation of the heat flux in the circumferential direction.

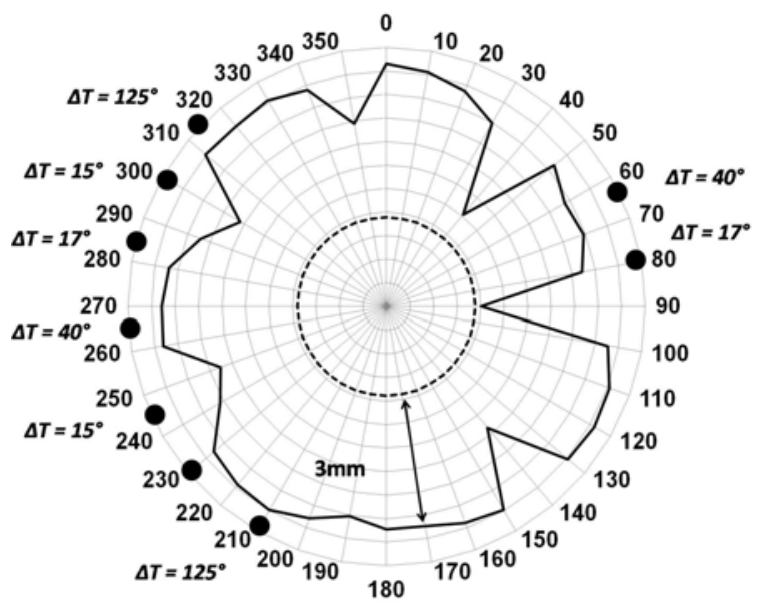

Figure 17. Circumferential wear of abradable coating and thermocouple variations at $100 \mathrm{~mm}$ from the frame.

In fact, due to test conditions, the temperature increase is mainly due to blade-casing contact (tests are performed under vacuum conditions). Heat production, occurring when the blade has a predominant bending motion, leads to thermal propagation mainly in the radial direction. In the first configuration, the thermal variation corresponds to the increase of the blade dynamic response between 880 and $920 \mathrm{~s}$ after the beginning of the test. It means firstly that frictional contact dissipation increases continuously during the second phase. Secondly, the long period of stabilized dynamic response of this second phase as the temperature increases means that wear and thermal expansion of the components (casing and blade) modify the blade-casing contact conditions. 
These slow variations finally lead to favorable blade casing contact conditions for an unstable blade vibratory behavior. This conclusion may be extended to the second configuration with various periods of dynamic responses separated by low dynamic behavior stages. The changes of the blade vibratory responses may correspond to a change of contact conditions. Remaining that during this test the blade is more constrained an assumption of interpretation is that the blade is successively excited by variable contact conditions. The blade is less and less constrained during the test due to the wear increase and thermal expansion of the components illustrated by various dynamic responses. Firstly 2 contacts per revolution corresponding to the initial ovalization may be explained by the predominance of $\mathrm{H} 2$. Then the stiffness of the blade submitted to contact forces may increase as shown by spectrum with a predominance of H7. Finally the changes of contact conditions with wear and thermal expansion attenuate the blade constraint leading to an unstable dynamic response coinciding with a 6 multiple of the rotation speed.

\section{CONCLUSION}

Experimental investigations of blade-casing interactions have been studied in order to highlight the mechanisms involved in the blade failure. Several tests were carried out with one blade longer than the others and equipped with strain gages. In each case the longer blade cracked by fatigue damage due to the excitation of the first blade bending mode. Blade stress time variation has shown that the blade diverged not directly but after more or less long periods with several stages of various dynamic responses. Unstable vibratory responses have been observed without blade damage. It appears that the dynamic behavior is strongly influenced by the bladed disk/casing contact.

It has been observed non uniform wear profile along the axial and circumferential directions. The number of wear lobes in the circumferential direction corresponds to the number of periods per revolution of the first blade bending mode. Moreover significant temperatures increases have been measured at the rear of casing. It has been shown that time variations of temperatures may be correlated with the different phases of the blade dynamic response. Note that the low conductivity of the coating material means that these temperatures are higher at the contact surface.

These analyses show that the thermal-mechanical phenomena at the contact have to be considered, i.e. wear and thermal dilatations of the components. It may explain why various stages of dynamic behavior are observed with different frequency spectrum of excitation. It illustrates the coupling between the dynamic behavior and the blade casing contact variations induced by thermomechanics and wear. Improving the analysis of this coupling needs additional numerical investigations about heat fluxes induced by the contact, deformations and consequences on the dynamic response.

\section{REFERENCES}

[1] Song, S.J. and Martinez-Sanchez, M., 1997, "Rotordynamic forces due to turbine tip leakage: Part I -
Blade scale effects". Journal of Turbomachinery, vol. 119, pp 695-703.

[2] Song, S.J. and Martinez-Sanchez, M., 1997, "Rotordynamic forces due to turbine tip leakage: Part II - Radius scale effects and experimental verification". Journal of Turbomachinery, vol. 119, pp 704-713.

[3] Sinha, S.K., 2004, "Dynamic characteristics of a flexible bladed-rotor with Coulomb damping due to tip-rub", Journal of Sound and Vibration, vol. 273 (4), pp. 875919.

[4] Jiang, J. and Ulbrich, H., 2000, "Dynamics and stability of rotor/stator systems with rubs", ASME turbo expo Munich Germany, 2000 GT-390.

[5] Edwards, S., Lees, A.W. and Friswell, I., 1999, "The influence of twisting on rotor/stator contact in rotating machinery", Journal of Sound and Vibration, vol. 225 (4), pp. 767-778.

[6] Ehrich, F.F., 1969, "the dynamic stability of Rotor/ Stator Radial Rubs in rotating machinery", Journal of Engineering for Industry, Vol. 91 (4), pp. 1025-1028.

[7] Ehrich, F.F., 1993, "Rotor whirl forces induced by the tip clearance effect in axial flow compressors", Journal of Vibration and Acoustics, vol. 115, pp 509-515.

[8] Goldman, P. and Muszynska, A., 1995, "Rotor to stator, rub-related, thermal/mechanical effects in rotating machinery", Chaos, Solitons and Fractals, vol. 5 (9), pp. 1579-1601.

[9] Bachschmid, N., Pennacchi, P. and Vania A., 2007, "Thermally induced vibrations due to rub in real rotors", Journal of Vibration, vol. 299, pp. 683-719.

[10] Faraoun H.I., Seichepine J.L., Coddet C., Aourag H., Zwick J., Hopkins N., Sporer D., Hertter R., 2006, "Modeling route for abradable coatings", Surface and coatins technology, vol. 200 (22-23), pp. 6578-6582.

[11] Seichepine, J.L., Albertazzi, S., Nardin, P., Coddet, C. and Sporer D., 2005, "Topological characterization of wear tracks in thermally sprayed abradable coatings", International Thermal Spray Conference (ITSC), Basel, Switzerland.

[12] Lugscheider, E., Zwick, J., Hertter, M. and Sporer D., 2005, "Control of Coating Properties of Abradable Seals by On-Line Process Diagnostics", International Thermal Spray Conference (ITSC), Basel, Switzerland.

[13] Maozhong, Y., Baiyun, H. and Jiawen H., 2002, "Erosion wear behavior and model of abradable seal coating", Wear, vol. 252 (1), pp. 9-15.

[14] Arnoult, E., Peseux, B. and Berthillier M., 1998, "Aircraft engine blades-casing contact study", International Conference of Computational Engineering Sciences, Atlanta, USA, vol. 2, pp. 1032-1037.

[15] Sutter, G., Philippon, S. and Garcin, F., 2006, "Dynamic analysis of the interaction between an abradable material and a titanium alloy", Wear, vol. 261, pp. 686-692. 